Cuadernos de Historia Contemporánea

ISSN: 0214-400X

http://dx.doi.org/10.5209/CHCO.54304

\title{
La violencia política durante la Segunda República
}

Fernando Jiménez Herrera ${ }^{1}$

Eduardo González Calleja y Rocío Navarro Comas (Eds.): La España del Frente Popular. Política, sociedad, conflicto y cultural en la España de 1936. Granada, Comares, 2011.

Eduardo González Calleja: En nombre de la autoridad. La defensa del orden público durante la Segunda República Española (1931-1936). Granada, Comares, 2014.

Eduardo González Calleja: Cifras cruentas. Las víctimas mortales de la violencia sociopolítica en la Segunda República Española (1931-1936). Granada, Comares, 2015.

José Luis Martín Ramos: El Frente Popular. Victoria y derrota de la democracia en España. Barcelona, Pasado y presente, 2016.

Eduardo González Calleja es un bien conocido historiador especializado, entre otros ámbitos, en la investigación sobre la teoría y la historia de la violencia política. Sus trabajos han supuesto en este campo un referente de análisis y estudio para el resto de historiadores e historiadoras que abordan esta temática en sus trabajos. En la presente nota bibliográfica se analizarán dos obras suyas, En nombre de la autoridad. La defensa del orden público durante la Segunda República Española (1931-1936), del año 2014, y Cifras cruentas. Las víctimas mortales de la violencia sociopolítica en la Segunda República Española (1931-1936) de 2015. Dos obras que han contribuido a la reformulación de hipótesis sobre la violencia política durante la Segunda República. Además, se estudiará en la presente nota otra obra La España del Frente Popular. Politica, sociedad, conflicto y cultural en la España de 1936, en este caso editada junto con Rocío Navarro Comas, profesora también en la Universidad Carlos III, como el propio González Calleja.

Encargándose este último de la revisión y edición, esta obra recoge las propuestas presentadas al Congreso Internacional La España del Frente Popular. Política, sociedad, cultura y conflicto en la España de 1936, celebrado en la Universidad Carlos III de Madrid, en Getafe, los días 15 y 16 de febrero de 2011. También forma parte de la presente nota la obra de José Luis Martín Ramos El Frente Popular. Victoria y derrota de la democracia en España, un breve ensayo donde su autor analiza de forma crítica los relatos sobre la Segunda República, extendiendo su cronología hasta la guerra civil española, más concretamente hasta el año 1938 (en referencia a la existencia del Frente Popular). Una obra que comparte espacio de análisis y cronología

Universidad Complutense de Madrid (España)

E-mail: fernandojimenez@ucm.es 
con las obras de Eduardo González Calleja analizadas en la presente nota, como La España del Frente Popular. Política, sociedad, conflicto y cultural en la España de 1936. Por lo tanto, se van a analizar a continuación cuatro obras que abordan como objeto de estudio diversas facetas y etapas de la Segunda República española, poniendo el foco de atención sobre la violencia política.

Como presentación de las obras que componen esta nota bibliográfica, se va a proceder a analizar primero el contenido de estas obras, para pasar después a valorar y poner en común las aportaciones de las mismas en sus respectivos campos. El orden de análisis será el cronológico, tomando como referencia la fecha de edición. Comenzamos con La España del Frente Popular. Política, sociedad, conflicto y cultural en la España de 1936, que, como se ha mencionado anteriormente, recoge los trabajos presentados en el Congreso que da el título al libro. La cronología de los trabajos recogidos, en general, se ciñe a febrero-julio de 1936, es decir, el periodo de gobierno del Frente Popular, desde el 16 de febrero, en que gana las elecciones, hasta el golpe de estado del 17 de julio. Sin embargo, hay trabajos que exceden esa cronología y la alargan hasta diciembre de ese mismo año o enero de 1937. El libro se divide en cuatro partes o capítulos, abordando cada uno un aspecto significativo. Por lo tanto, hay un primer capítulo referido a los partidos políticos y su radicalización, el análisis del proceso constituyente y el cambio de poder a escala local por la victoria del Frente. Un segundo capítulo recoge aspectos relacionados con la protesta, la conflictividad y la violencia política. El tercero va dedicado a la esfera internacional. Y finalmente, el cuarto analiza aspectos de la vida cultural durante el Frente Popular: deporte, periódicos, arte o cine. Gracias a la diversidad de ponencias e intervenciones se abordan aspectos muy dispares, lo que hace que este libro sea una obra completa en cuanto a temáticas.

En este trabajo se pueden encontrar contenidos relacionados con la escala micro y estudios locales, así como enfoques más generales o de carácter macro. En el caso de los estudios locales son muy variados, ofreciendo la posibilidad de conocer diversas realidades en el Estado español durante la primera mitad del año 1936. Se comienza examinando los significados del concepto Frente Popular y sus interpretaciones en el caso español, para, a continuación, centrarse en tópicos y mitos y someterlos a crítica. Al día de hoy, desde diversos sectores sociales, se sigue creyendo responsable al Gobierno del Frente Popular y a este periodo de la situación que derivó en un golpe de estado que produciría la cruenta y larga guerra civil. Como bien recogen las comunicaciones de este trabajo, esta afirmación pertenece más al mito que a la realidad. Es un argumento utilizado por los militares sublevados como forma de legitimar su golpe de estado, terreno que fueron allanando los medios conservadores partidarios del golpe de estado con la intención de recuperar el poder perdido en las urnas. La actitud tomada por estos colectivos durante la primavera de 1936 frente al Estado provocó -entre otros factores como los niveles de protesta o la preparación de las fuerzas del orden ante las mismas, tema que aborda Eduardo González Calleja en su obra En el nombre de la autoridad, que se analizará a continuación-, que las relaciones sociales se deteriorasen. No obstante, en este otro trabajo de edición compartida, la idea principal o hilo conductor de las comunicaciones que la componen, es que se deje por fin de entender el periodo como la culminación del fracaso del proyecto republicano, y se pase a valorar el conjunto de medidas y actitudes tomadas ante la crisis que se planteó en esos meses de 1936. 
El primer apartado se centra, pues, en el análisis de los partidos políticos y las movilizaciones de los mismos y de sus bases durante el periodo electoral de febrero de 1936. Movilizaciones que llevaron a la ocupación de espacios públicos como la calle. Pero no solo se estudia la movilización, sino que también se analiza el discurso político imperante entre los diversos sectores sociales. El lenguaje como transmisor de emociones y sentimientos, como forma de aunar voluntades, de convencer, atemorizar, movilizar, y como generador de ideas. Sin dejar de lado aspectos como la violencia o la radicalización interna a esa misma movilización y al lenguaje. En las ponencias relacionadas con la movilización, la conflictividad y la violencia sociopolítica son los principales elementos relacionados con las otras dos obras de Eduardo González Calleja que se analizan en esta nota bibliográfica. Los ensayos se centran en la violencia, la movilización y la conflictividad que se produjo a lo largo de todo el gobierno del Frente Popular, ya no solo, como en el caso anterior, durante el proceso electoral. Analizan el ambiente, generalmente tildado de terrorífico, en el sentido de persecución política como sinónimo de caos o desorden, responsabilizando de esta supuesta situación a los colectivos de izquierdas y al gobierno, y eludiendo o edulcorando la responsabilidad de los grupos conservadores en este tipo de situaciones. Mostrando así, en fin, un mito que envuelve a este periodo, definiéndolo como preparativo para la guerra civil, y siendo la base de la violencia revolucionaria que se desató en las zonas que permanecieron fieles al Gobierno. En resumen, uno de los objetivos más relevantes de este apartado es el análisis cuidadoso de la violencia, estudiando minuciosamente motivaciones y situaciones que ayuden a distanciarla de la violencia en la guerra y con ello, a desligarla de esta y de su ejercicio por parte de las fuerzas revolucionarias.

En el siguiente apartado se estudia la dimensión internacional de la primavera de 1936, principalmente el propio fenómeno de la constitución de un Frente Popular, herramienta política ideada por los comunistas ante el éxito de Hitler en Alemania, como forma de contrarrestar el poder de fuerzas fascistas en otros países europeos, a través de la unidad de las izquierdas. Aunque fuese una idea comunista, eso no quiere decir que el Frente Popular en España u otros países de entornos cercanos estuviesen dirigidos por dicha organización política. En la España de la primavera de 1936, el Partido Comunista Español tenía un papel minoritario en referencia al resto de fuerzas obreras del país, sobre todo frente a anarquistas y socialistas. El excesivo protagonismo que le otorgaron sectores mediáticos de las fuerzas conservadoras antes del golpe de estado, y, una vez producido el mismo, por parte de la propaganda sublevada, fueron totalmente infundadas (en el caso de la guerra, su uso respondió a un deseo de legitimar un golpe de estado contra un Gobierno legítimamente elegido en las urnas). Este apartado se centra también en las relaciones internacionales del gabinete frentepopulista con el resto de fuerzas europeas y con organismo internacionales como el Socorro Rojo Internacional (SRI). Finalmente, el libro se cierra con un cuarto apartado destinado al estudio de los aspectos culturales y de vida cotidiana durante el Gobierno del Frente Popular. Estudios sobre la prensa y su repercusión, sobre el arte, el cine, pero también el deporte como elementos destacados en la configuración de mentalidades y corrientes de opinión, y en la transmisión de mensajes que influían en el imaginario colectivo de sus lectores y lectoras a la hora de generar opinión.

Esa primera obra editada por Eduardo González Calleja y Rocío Navarro, enlaza a la perfección con la titulada En nombre de la autoridad. La defensa del orden 
público durante la Segunda República Española (1931-1936) del propio Eduardo González Calleja. Ambas abordan, como indicamos, el tema de la protesta, si bien aquí se aborda toda la Segunda República, incluyendo naturalmente el Frente Popular. La protesta fue la que ocasionó más muertes a causa de la violencia sociopolítica durante la Segunda República, y la hipótesis principal de este trabajo es que, al advenimiento de la república, la ciudadanía accedió a espacios y formas de protesta antes vetados, por lo que, en numerosos casos carecían de experiencias previas que pautaran esas protestas y facilitaran su gestión. Asimismo, los cuerpos de orden público y vigilancia estaban curtidos en experiencias previas represivas más que preventivas, por lo que consideraron desacato y provocación actos de manifestantes o huelguistas, lo que derivó en general en cargas y represión como forma de control. Por otro lado, el Gobierno republicano, en su primera experiencia de mandato, se encontró ante una situación incómoda: el obligado uso de las fuerzas del orden público para controlar la protesta y mantener bajo control el poder recientemente adquirido. Así, el objetivo de Eduardo González Calleja es, en palabras del propio autor, "estudiar un aspecto concreto de las distintas subculturas políticas de la época republicana... con la violencia sociopolítica: los instrumentos y las estrategias de defensa del orden público" (p. 7).

Habiendo explicado en la introducción y en el primer capítulo el significado del término "orden público" y su interpretación en España, y los antecedentes de los cuerpos que compusieron las fuerzas del orden público durante la Restauración, González Calleja analiza, a lo largo de los seis capítulos restantes, las relaciones entre los distintos gobiernos de la Segunda República, que favorecieron o no, dependiendo del signo político, leyes restrictivas, apoyos a las fuerzas del orden y los poderes tradicionales, restricción de armamento para diversos segmentos de la población y obtención de armas de forma legal para personas pudientes -representantes de los poderes tradicionales-, las fuerzas del orden y la evolución de la protesta, mostrando que la falta de preparación de las fuerzas del orden, unida a su deslealtad al gobierno (principalmente en el caso de la Guardia Civil, como consecuencia de su experiencia en la Primera República, que evocaba en ellos caos y anarquía), harían que se recurriese a la violencia como forma de gestión y control de la protesta. En este contexto, y ante la evidente necesidad de modernización de estos colectivos, se crearía la Guardia de Asalto, un cuerpo que los gobernantes republicanos de izquierdas supusieron fiel al régimen del 14 de abril, que recibió preparación y material adecuado para enfrentarse, de nuevo, a otras situaciones de protesta.

Los gobiernos de izquierdas, para la constitución de este cuerpo, emularon a las democracias occidentales, Francia e Inglaterra, quienes formaron cuerpos especiales para controlar las protestas populares tras la Gran Guerra. Por lo tanto, y como afirma Eduardo González Calleja, desde los gobiernos de izquierdas hubo una voluntad por hacer un uso eficiente de las fuerzas del orden, adaptándolas al nuevo contexto democrático que toleraba una gran variedad de formas de protesta. No obstante, también se dictaron leyes que blindaran la República, como forma de protegerla frente a los ataques promovidos tanto desde sectores conservadores como obreros, monárquicos y anarquistas respectivamente. Estas leyes permitieron la vulneración de derechos fundamentales que otorgaba la constitución de 1931 a la ciudadanía.

El orden público, como bien concluye el autor, fue un problema de estabilidad para la Segunda República, con un mínimo de 2.624 víctimas mortales a lo largo de los cinco años de vigencia del régimen del 14 de abril. El mayor número de las 
víctimas, en torno a las 2.000 , se produjeron por enfrentamientos entre la policía y los protagonistas de la protesta, en su inmensa mayoría manifestaciones y huelgas pacíficas. Las cifras que aparecen a lo largo de la obra y que ofrecen el balance final (mínimo) son fruto del estudio detallado y exhaustivo, por parte del autor, de diversas fuentes documentales y hemerográficas para todas las regiones que formaban el estado español durante la Segunda República. Indica Eduardo González Calleja que esta cifra no es definitiva, pues plantea la problemática que ofrecen las fuentes en referencia a Asturias en la revolución de octubre de 1934. Debido a la censura, no se exponen en los medios balances de cifras fiables. Estos balances solo se ofrecen de forma oficial y muy limitada, lo que dificulta la labor del historiador y la historiadora a la hora de reconstruir el impacto real que tuvo la labor represiva sobre la población civil en la zona. Sin embargo, las cifras que se recogen a lo largo de la obra en referencia al resto de regiones, son fruto de un exhaustivo proceso de análisis y crítica de fuentes, lo que le otorga a este estudio un valor especial, por su solidez académica y rigor histórico. Otro aspecto que fortalece a esta investigación es el proceso de comparación con otros trabajos que fomentan la discusión sobre las cifras totales de este género de violencia. Como escribe el autor, uno de los factores que contribuyó a que se diesen tales niveles de violencia por parte de las fuerzas del orden hacia la población civil movilizada fue que "la gestión cotidiana de los derechos de los ciudadanos no se hizo de forma extensiva en nombre de la democracia, sino de un modo restrictivo en nombre de la autoridad" (p. 326).

La última obra de Eduardo González Calleja que compone esta nota bibliográfica se titula Cifras Cruentas. Las víctimas mortales de la violencia sociopolítica en la Segunda República Española (1931-1936). Dentro de las tres obras analizadas, ésta es la más expositiva y cuantitativa, frente a las dos anteriores que son más analíticas e interpretativas. No obstante, esta obra no se limita a recoger y exponer todos los casos de violencia sociopolítica que tuvieron resultado de muerte, sino que aborda, además del número, la identidad y las circunstancias de las víctimas de la violencia social y política del periodo, y el carácter intencionado o fortuito de la acción, e incluso, el espacio donde se produce ésta. Otro de los aspectos tratados es la repercusión y la tergiversación llevada a cabo por medios conservadores en su propio provecho, para obtener rédito político describiendo la situación como un caos e identificando a los violentos con los colectivos de izquierda en detrimento de los violentos de derechas. Serán objetos de estudio de este trabajo, tanto actos de violencia colectiva como individual, siempre que su autor considere que, detrás de estos actos, existió una intencionalidad social o política. Se recogen así algunos datos relacionados con la violencia ejercida por la autoridad y las fuerzas del orden público ya mencionados en En nombre de la autoridad, del mismo autor. Otro de los problemas que se examinan aquí es el relacionado con los sucesos represivos sobre la revolución de octubre en Asturias por parte de las autoridades militares enviadas para imponer su control sobre los revolucionarios. Al estar vigente el estado de guera y la censura, no se permitió a los medios hacerse eco de las actividades represivas de los militares sobre la población civil, por lo que se carecen de recuentos fiables y datos personales sobre las víctimas de esta represión.

A lo largo de este trabajo se afirma el carácter heterogéneo en cuanto al ámbito, la tipología y los protagonistas de la violencia sociopolítica, además de caracterizarse por sus grandes dosis de espontaneidad, con una mayor organización en los centros urbanos respecto a las zonas rurales. Los resultados le permiten al autor desmentir 
la existencia, a lo largo de la Segunda República, de una violencia revolucionaria organizada por la izquierda para tomar el poder, principal argumento de los militares sublevados el 17 de julio de 1936 para legitimar el golpe de estado. También le autorizan a detectar un alto predominio de formas paramilitares en los grupos de extrema derecha, principalmente a partir de la victoria del Frente Popular en febrero de 1936.

En referencia a la responsabilidad, Eduardo González Calleja confirma que el colectivo que más violencia sociopolítica con resultados de muerte ejerció fueron las fuerzas del orden público, principalmente la Guardia Civil. Esto se debió a su falta de medios y preparación para inscribirse en un contexto democrático. Siguiendo las argumentaciones del autor, la violencia sociopolítica durante el periodo republicano debe interpretarse como "la manifestación inmediata y notoria de un estado de agitación social y de lucha política que se conecta con la dinámica de enfrentamiento entre proyectos reaccionarios, reformistas y revolucionarios... que alcanzó su momento de mayor intensidad conflictiva durante la dramática andadura de la democracia nacida el 14 de abril de 1931" (p. 307). Por ello, la violencia que surge a partir del golpe de estado nada tiene que ver, en general, con esa otra violencia, ya que sus lógicas son diferentes. Ni tampoco es lícito considerar esta violencia como causa de la guerra civil.

La última obra que compone esta nota bibliográfica es el trabajo de José Luis Martín Ramos, El Frente Popular. Victoria y derrota de la democracia en España. En este ensayo el autor se centra en el análisis y estudio del Frente Popular como alianza frente al ascenso del nazismo. A través de sus cinco capítulos, contradice diversas interpretaciones sobre el periodo y el gobierno frentepopulista originadas en sectores conservadores de la sociedad, como forma de ataque y deslegitimación durante la Segunda República y la guerra civil, interpretaciones que se mantuvieron durante la dictadura franquista, perviviendo todavía al día de hoy entre diversos sectores sociales que buscan rentabilidad política. Aunque el autor pudiere parecer tendencioso, ya que él mismo define su postura como beligerante, la argumenta y defiende con sus muchos años de investigación y la consulta de innúmeras fuentes accesibles al público. A lo largo de la obra, si alguna hipótesis no ha podido ser refutada por la documentación, el autor advierte de ello a los lectores.

No encontramos así ante un ensayo de carácter divulgativo, sin perder el rigor científico, cuyo objeto, en palabras del autor, es "el Frente Popular en España, su triunfo democrático en las urnas, indiscutible por más que se pretendan retorcer los números; el desarrollo democrático de su programa, mediante la acción ejecutiva del gobierno" (pp. 8-9). Una vez analizado el contexto y los objetivos con los que nació el Frente Popular, José Luis Martín Ramos analiza la evolución del mismo y su materialización en las urnas para, finalmente, acabar su relato explicando los cambios que sufrió el régimen político.

Por lo tanto, hay dos hilos conductores que ligan estas cuatro obras y permiten un análisis conjunto. Por un lado el contexto histórico, la Segunda República, y por otro, el que en todas se realizan estudios sobre la violencia, ya sea esta fomentada por un periodo de elecciones y cambios de gobiernos locales y nacionales (La España del Frente Popular. Política, sociedad, conflicto y cultural en la España de 1936), por las fuerzas del orden público: En nombre de la autoridad. La defensa del orden público durante la Segunda República Española (1931-1936), por enfrentamientos de carácter sociopolítico entre las bases de los partidos y frente a las autoridades (Cifras cruentas. Las víctimas mortales de la violencia sociopolitica en la Segunda 
República Española (1931-1936)), o durante el proceso electoral de 1936 y ante el inicio de la guerra civil (El Frente Popular. Victoria y derrota de la democracia en España). Al compartir estos espacios de análisis y estudio, contribuyen unos a otros a poder comprender mejor la Segunda República española, sirviendo mutuamente de contextualización, no solo por abordar el mismo régimen político, sino también por su temática, la violencia política. Una violencia que fue ejercida por las fuerzas del orden (En el nombre de la autoridad), por colectivos políticos (Cifras cruentas), y por ambas partes (La España del Frente Popular y El Frente Popular. Victoria y derrota de la democracia en España). Y una violencia interrelacionada, lo que ayuda a explicar el contexto y desenlace posterior.

Las cuatro obras analizadas suponen una renovación en cuanto a perspectivas de análisis sobre política, violencia sociopolítica y fuerzas del orden público durante la Segunda República Española y, principalmente, el periodo del Frente Popular. Unas aportaciones de relevancia que nos permiten contrarrestar los mitos que perviven en nuestra sociedad, y entender y comprender de una forma más exhaustiva el contexto estudiado. Mitos como, por ejemplo, el de la radicalización exclusiva de la izquierda frente a una derecha víctima y victimizada, dando lugar a la invisibilización intencionada de la violencia y radicalización de los colectivos conservadores. Otro mito que pervive al día de hoy es la consideración del Frente Popular como un caos que condujo de forma inevitable hacia la contienda civil, lo que legitima el golpe de estado militar contra un gobierno legítimo refrendado en las urnas. Estos argumentos, junto a otros relatos mitificados sobre el periodo, son aquí puestos en cuestión en un conjunto de relatos que pretenden ofrecer un análisis riguroso y detallado, fruto de años de trabajo e investigación. 